\title{
On the Approach to the Final Aperiodic Regime in Maps of the Interval
}

\author{
J. Dias de Deus and J. Taborda Duarte
}

CFMC-Instituto Nacional de Investigação Cientifica, P-1699 Lisboa Codex, Portugal

\begin{abstract}
We present a global approach to the final aperiodic regime in maps of the interval displaying a simple pattern with similarities to the Feigenbaum scheme.
\end{abstract}

\section{Introduction}

It is well known that complex dynamical behaviour can be generated by maps of the interval into itself. In particular the mechanism of period doubling bifurcation by functional iteration provides a procedure to achieve, starting with simple periodic systems, aperiodic regimes. For functions $f_{\mu}(x)$ belonging to $C^{2}[-1,1]$, even and convex, depending on a parameter $\mu$ in $[0,1]$ such that (for every $x$ ) $f_{0}(x)=1, f_{1}(0)=-1$ and $f_{\mu}( \pm 1)=1$, numerical and analytic studies have revealed remarkable properties in the approach of the aperiodic regime by period doubling bifurcation. Let us briefly summarize here these properties [1]:

i) Each orbit of period $2^{n}$ has in $\mu$ a region $I_{n}$ of stability above which it ceases to be stable originating (by bifurcation) an orbit of period $2^{n+1}$.

ii) The sequence $\left(\mu_{n}\right), n=0,1,2, \ldots$, where $\mu_{n}$ is the value of $\mu$ for which the orbit of period $2^{n}$ is super-stable, is strictly increasing with limit $\mu_{\infty}<1$.

iii) As $n \rightarrow \infty$ the sequence $\left(\mu_{n}-\mu_{n-1}\right)$ converges geometrically, i.e.,

$$
\lim \frac{\mu_{n}-\mu_{n-1}}{\mu_{n+1}-\mu_{n}}=\delta \text {. }
$$

iv) The Feigenbaunm ratio $\delta$ only depends on the behaviour of $f_{\mu}(x)$ at the critical point $x=0$ (universality).

v) The iterated functions $f_{\mu_{n}}^{2^{n}}(x)$ and $f_{\mu_{n+1}}^{2^{n+1}}(x)$ are similar and related by a scaling transformation which determines a second Feigenbaum ratio $\alpha$.

In the present paper we consider the approach to aperiodic behaviour from a more global point of view. After the first Feigenbaum window in $\mu,\left[0, \mu_{\infty}\right]$, other windows of periods $N 2^{n}, N=3, \ldots, n=0,1, \ldots$, as well as regions of aperiodicity follow. The final aperiodic limit is achieved for $\mu \rightarrow 1$. The various regions of stability for the different periods, and the aperiodic regions are all mixed up in a 
very complicated "chaotic" manner. However it is possible to show that a regular pattern, bearing close analogy to the Feigenbaum pattern i) to v) above, emerges in the approach of the final aperiodic limit as $\mu \rightarrow 1$. More precisely we prove that ${ }^{1}$ :

$\left.i^{\prime}\right)$ The region of $\mu$ in $[0,1]$, where the orbit of period $k, k=1,2,3, \ldots$, definitely ceases to be stable appears before the similar region for the orbit of period $k+1$ and, for the super-stable points,

$$
\mu_{k}<\mu_{k+1}
$$

ii') The sequence $\left(\mu_{k}\right)$ is strictly increasing with limit 1 .

iii') As $k \rightarrow \infty$ the sequence $\left(\mu_{k}-\mu_{k-1}\right)$ geometricaly converges

$$
\lim \frac{\mu_{k}-\mu_{k-1}}{\mu_{k+1}-\mu_{k}}=\Delta
$$

iv $^{\prime}$ ) The ratio $\Delta$ only depends on the behaviour of $f_{\mu}(x)$ at $x=1$ (universality) and

$$
\Delta=f_{1}^{\prime}(1) \text {. }
$$

$\left.\mathrm{v}^{\prime}\right)$ The iterated functions $f_{\mu}^{k}(0) \equiv g(k, \mu)$ in the $k \rightarrow \infty$ limit scale

$$
g(k, \mu) \underset{k \rightarrow \infty}{\rightarrow} h(X), \quad X \equiv \Delta^{k}(1-\mu) .
$$

In particular, for $(1-\mu)=\Delta(1-\tilde{\mu})$, one has

$$
f_{\mu}^{k}(0) / f_{\tilde{\mu}}^{k+1}(0) \underset{k \rightarrow \infty}{\rightarrow} 1 .
$$

Clearly, the statements $i^{\prime}$ ) to $v^{\prime}$ ) to be proven in the next section closely resemble the statements i) to v) valid in Feigenbaum scheme. There are, at the same time, essential differences. While Feigenbaum locally explores the development of aperiodicity by period doubling, within a given window we explore the whole range of $\mu,[0,1]$, and look for the final establishment of aperiodic regime by successive and definite disappearance of stable orbits of increasing periodicity. For $n=1$ the super-stable Feigenbaum point is the final super-stable point for orbits of period 2, but the super-stable Feigenbaum for $n=2$ is not the ultimate super-stable point of period 4 and is then not included in our sequence $\mu_{k}$. In general the super-stable points associated to the harmonics do not belong to the sequence $\mu_{k}$.

The numbers $\mu_{k}$ divide the interval $[0,1]$ in a particular way [2]. In each interval $\left(\mu_{k}, \mu_{k+1}\right)$ the kneading sequences have in common the first $k+1$ terms and this is enough to characterize the dynamics in that interval as being topologically not equivalent to the dynamics in any other interval. To each interval we can associate a finite sequence $S_{k}$. The point $\mu_{k}$ corresponds to the transition from $S_{k}$ to $S_{k+1}$. The approach to the final aperiodic regime corresponds to the limit of $S_{k}$ as $k \rightarrow \infty$. This is discussed in Sect. 3 .

Finally we would like to mention that the sequence $\mu_{k}$ has been previously noticed, in a different context and for a specific example, by Guckenheimer [3].

1 The proof of $\left(i i^{\prime}\right)-v^{\prime}$ ) is formal rather than completely rigorous 


\section{The Approach to the Final Aperiodic Limit: The Sequence of $\left(\mu_{k}\right)$}

Consider $I=[-1,1]$ and let $C_{0}$ be the set of functions $f: I \rightarrow I$ belonging to $C^{3}(I)$ such that

i) $f(-1)=f(1)=1$.

ii) $f$ has a single local minimum at $x=0 ; f$ is strictly decreasing on $[-1,0]$ and strictly increasing on $[0,1]$.

iii) For every $x \in I, f^{\prime \prime}(x)>0$.

Let us take a parameter space $J=[0,1]$ and define a map $J \rightarrow C_{0}$ defined by $\mu \rightarrow f_{\mu}$. We can then prove the following:

Theorem 1. Let $\mu \rightarrow f_{\mu}$ be differentiable and

a) $f_{1}(0)=-1, f_{0}(x) \equiv 1$.

b) The induced map $f_{(1)}: J \rightarrow I$ defined by $f_{(1)}(\mu)=f_{\mu}(0) \equiv f(\mu, 0)$ is strictly decreasing:

Then if we consider $\mu_{k}=\sup \left\{\mu \in J \mid f_{\mu}^{k}(0)=0\right\}$, the sequence $\left(\mu_{k}\right)$ is strictly increasing.

Proof. We introduce the following notation. Define $f_{(k)}: J \rightarrow I$ by $f_{(k)}(\mu)=f_{\mu}^{k}(0)$. Then observe that for each $k>1$ we have $f_{(k)}(1)=1$. As $f_{(k)}\left(\mu_{1}\right)=0$ we must have $\mu_{k} \geqq \mu_{1}$. By computing the derivative $f_{(k)}^{\prime}\left(\mu_{1}\right)$ we get $f_{(k)}^{\prime}\left(\mu_{1}\right)=\frac{\partial f}{\partial \mu}\left(\mu_{1}, 0\right)<0$. So there must be a zero of $f_{(k)}$ in $\left(\mu_{1}, 1\right)$ which means that $\mu_{k}>\mu_{1}$. Then by assumption b) we have $f_{(1)}\left(\mu_{k}\right)<f_{(1)}\left(\mu_{1}\right)$. But

$$
f_{(k+1)}\left(\mu_{k}\right)=f_{\mu_{k}}\left(f_{(k)}\left(\mu_{k}\right)\right)=f_{\mu_{k}}(0)=f_{(1)}\left(\mu_{k}\right)<0 .
$$

There must be $\tilde{\mu} \in\left(\mu_{k}, 1\right)$ such that $f_{(k+1)}(\tilde{\mu})=0$. This implies that $\mu_{k+1} \geqq \tilde{\mu}>\mu_{k}$ as we wanted to prove.

This sequence $\left(\mu_{k}\right)$ is the basis of our claim of introducing a regularity pattern to the complicated behaviour of functions $f_{(k)}$.

Proposition 2. Let $\mu \rightarrow f_{\mu}$ be a map verifying the assumptions of Theorem 1 and moreover, for each $x \in I f_{\mu}(x)=\mu P(x)+Q(x)$ for any $\mu \in J$. Then for every $k>1$ and any $\mu \in\left(\mu_{k}, 1\right)$ we have $f_{(k)}^{\prime}(\mu) \geqq 0$.

Proof. This result will be proved by induction. Remark that $f_{(2)}^{\prime}\left(\mu_{2}\right) \geqq 0$ for otherwise $\mu_{2}$ would not be the last zero of $f_{(2)}$. We can write, making use of a), $f_{\mu}(x)=\mu P(x)+1$ with $P(0)=-2$ and $P( \pm 1)=0$. Hence there comes $f_{(2)}^{\prime \prime}(\mu)=$ $-4 P^{\prime}(1-2 \mu)+4 \mu P^{\prime \prime}(1-2 \mu)$. As $\mu_{1}=\frac{1}{2}$, if we take $\mu>\mu_{2}>\mu_{1}$ we have $P^{\prime}$ $(1-2 \mu)<0$ and this enables us to conclude that $f_{(2)}^{\prime \prime}(\mu)>0$. By mean value theorem on the interval $\left(\mu_{2}, 1\right)$ we may assert that $f_{(2)}^{\prime}(\mu)>f_{(2)}^{\prime}\left(\mu_{2}\right)>0$. By induction it is now easy to prove using the same arguments that $f_{(k+1)}^{\prime}(\mu)>0$ for any $\mu \in\left(\mu_{k+1}, 1\right)$.

Remark. With the assumption of Proposition 2 we may assert that $f_{(k)}$ restricted to the interval $\left(u_{k-1}, 1\right)$ has only one root and a more general family $f_{\mu}(x)=g(\mu) P(x)$ +1 could be used.

We next prove a lemma which will be useful in subsequent computations. 
Lemma. Let $\mu \rightarrow f_{\mu}$ be a map satisfying properties as stated in Proposition 2. Then the sequence $\left(\mu_{k}\right)$ converges to $\mu_{L}=1$.

Proof. Reasoning by contradiction we assume that $\mu_{L}$, which exists since $\left(\mu_{k}\right)$ is monotone and bounded, is less than one. As $\mu_{L}>\mu_{p}$ for any $p$ we claim that $\left(f_{k}\left(\mu_{L}\right)\right)_{k>1}$, is a strictly decreasing sequence converging to zero.

As $f_{(k)}\left(\mu_{L}\right)=\mu_{L} P\left(f_{(k-1)}\left(\mu_{L}\right)\right)+1$ we notice by taking limits as $k \rightarrow \infty$ that $0=\mu_{L} \cdot P(0)+1$, i.e., $P(0)=-\frac{1}{\mu_{L}}$. As $\mu_{L}>\frac{1}{2}$ this contradicts the fact that $P(0)=-2$. Then we must have $\mu_{L}=1$.

Theorem 2. Let $\mu \rightarrow f_{\mu}$ be a map as stated before. Assume now in addition that $f_{\mu}$ is an analytic function. Then $\Delta_{k}=\frac{\mu_{k}-\mu_{k-1}}{\mu_{k+1}-\mu_{k}}$ is a convergent sequence and $\lim _{\mu \rightarrow \infty} \Delta_{k}=\Delta=f_{1}^{\prime}(1)$.

Proof. For each $k \geqq 2$ and any $\mu \in[0,1]$ we have

$$
f_{(k)}(\mu)=f_{\mu}\left(f_{(k-1)}(\mu)\right) .
$$

Then as $f_{\mu}(1)=1$ for any $\mu \in[0,1]$ we can write

$$
f_{(k)}^{\prime}(1)=f_{1}^{\prime}(1) f_{(k-1)}^{\prime}(1) \text {, }
$$

which implies $f_{(k)}^{\prime}(1)=\alpha_{1} \Delta^{k}$ for some constant $\alpha_{1}$.

We expand now at $\mu=1$ the function $f_{(k)}$,

$$
f_{(k)}(\mu)=\sum_{n} \frac{f_{(k)}^{(n)}(1)}{n !}(-1)^{n}(1-\mu)^{n},
$$

and estimate the asymptotic behaviour of the derivatives $f_{(k)}^{(n)}(1)$ when $k \rightarrow \infty$. As a consequence of $f_{\mu}(1)=1$ and $f_{(k)}^{\prime}(1)=\alpha_{1} \Delta^{k}, \Delta>1$, we shall see, that

$$
f_{(k)}^{(n)}(1) \sim \alpha_{n} \Delta^{n k} \quad(k \rightarrow \infty),
$$

where $\alpha_{n}$ is a constant $\left(\alpha_{n}=0\right.$ for some $n>2$ is possible but not relevant for the asymptotic behaviour). Omitting lower order terms in $\Delta$ we have

$$
\begin{aligned}
f_{(k)}^{(n)}(1) & \sim\left[f_{1} \circ f_{(k-1)}\right]^{(n)}(1) \\
& \sim \sum_{\left(r_{1}, \ldots, r_{n}\right) \in J_{n}} a_{r_{1}} \ldots r_{n}\left(f_{(k-1)}^{(1)}(1)\right)^{r_{1}} \ldots\left(f_{(k-1)}^{(n)}(1)\right)^{r_{n}},
\end{aligned}
$$

where $J_{n}=\left\{\left(r_{1}, \ldots, r_{n}\right) \in N^{n} \mid \sum_{i=1}^{n} i r_{i}=n\right\}$ and, for each $\left(r_{1}, \ldots, r_{n}\right), a_{r_{1} \ldots r_{n}}$ is constant. The estimate $(*)$ is then obtained by induction on $n$.

Taking as variable $X=\Delta^{k}(1-\mu)$, it is clear that $f_{(k)}$ can be asymptotically represented by a scaling function $h(X)^{2}$ which implies that $\lim _{k} \Delta^{k}\left(1-\mu_{k}\right)=X_{0} \neq 0$.

It is then obvious that $\lim _{k \rightarrow \infty} \frac{\mu_{k}-\mu_{k}-1}{\mu_{k+1}-\mu_{k}}=\Delta=f_{1}^{\prime}(1)$. 


\section{How the Sequence $\left(\mu_{k}\right)$ Organizes the Approach to the Final Aperiodic Regime}

To a differentiable one-dimensional map $f: I \rightarrow I$ with just one critical point at $x=0$, which is a minimum, can be associated a sequence $\gamma(f)=\left(a_{n}\right)_{n \geqq 0}$ on a set $E=\left\{c, I_{0}, I_{1}\right\}$, called the kneading sequence of $f$ and defined in the following way:

$$
a_{0}=c \text { and } a_{n}=\left\{\begin{array}{l}
c \text { if } f^{n}(0)=0, \\
I_{0} \text { if } f^{n}(0)<0, \\
I_{1} \text { if } f^{n}(0)>0
\end{array}\right.
$$

We consider the family $f_{\mu}$ of Sect. 2 and call $J_{k}$ the open interval $\left(\mu_{k}, \mu_{k+1}\right)$ for $k \geqq 1$ and $J_{0}=\left[0, \mu_{1}\right]$.

Theorem 3. Let $\mu \in J_{k}$ and $\gamma\left(f_{\mu}\right)=\left(a_{n}\right)_{n \geqq 0}$ be the kneading sequence of $f_{\mu}$. Then

i) If $k \geqq 1 \quad a_{n}=\left\{\begin{array}{l}c \text { for } n=0, \\ I_{0} \text { for } n \in\{1, k+1\}, \\ I_{1} \text { for } 1<n \leqq k .\end{array}\right.$

ii) If $k=0$ then $a_{n}=I_{1}$ for all $n>1$ and $a_{0}=c$.

Proof. 1. If $k=0$ and $\mu \in\left(0, \mu_{1}\right)$, we infer $f_{\mu}(0)>0$ and as $f_{\mu}$ is increasing on $(0,1)$ we conclude that for all $k \geqq 1 f_{\mu}^{k}(0)>0$.

2. If $k \geqq 1$ we know that $f_{\mu}(0)<0$ and $f_{\mu}^{k+1}(0)=f_{(k+1)}(\mu)<0$ for $\mu \in\left(\mu_{k}, \mu_{k+1}\right)$. The fact that $\mu>\mu_{k}>\mu_{k-1}>\ldots>\mu_{2}$ implies that we have $f_{\mu}^{2}(0), \ldots, f_{\mu}^{k}(0)$ all greater than zero.

We observe that the kneading sequences associated to $f_{\mu_{\mathrm{k}}}$ are $(C, I_{0}, \underbrace{I_{1}, \ldots, I_{1}}_{k-2}$, $C, I_{0}, I_{1}, \ldots, I_{1}, C$, etc...).

This observation has a consequence.

Corollary. If $\mu \in J_{k}$ and $\tilde{\mu} \in J_{l}$ then $f_{\mu}$ is not topologically equivalent to $f_{\tilde{\mu}}$.

The proof is obvious once we remark that two topological equivalent maps must have the same kneading sequences. The kneading sequences associated to $f_{\mu}$ for $\mu \in J_{k}$ for some $k$ may be very different but they all share the same $(k+1)$ first terms. In this sense we characterize by this finite sequence $S_{k}$ the interval $J_{k}$. The transition from $S_{k}$ to $S_{k+1}$ is done by replacing the last $I_{0}$ by a $I_{1}$ and adding a final $I_{0}$.

Now if we look at the final aperiodic regime, which corresponds to $\mu=1$, it has the kneading sequence $S_{\infty}=\left(C, I_{0}, I_{1}, I_{1}, \ldots\right)$. The sequence $S_{\infty}$ appears as a "limit" of the finite sequences $S_{k}$ as $k \rightarrow \infty$.

Acknowledgement. We would like to thank Prof. Ruelle for encouragement and for several critical remarks.

2 This step is formal rather than completely rigorous because the existence of the limits of the Taylor coefficients of a function does not imply the existence of the limit of the function. We believe however that the conclusion of our argument is correct 


\section{References}

1. Feigenbaum, M. J. : The Transition to aperiodic behaviour in turbulent systems. Commun. Math. Phys. 77, 65-86 (1980)

2. Metropolis, M., Stein, M. L., Stein, P. R.: On finite limit sets for transformation on the unit interval. J. Comb. Theory (A) 15, 25-44 (1973)

3. Guckenheimer, J.: Sensitive dependence on initial conditions for one dimensional maps. Commun. Math. Phys. 70, 133 (1979)

Communicated by D. Ruelle

Received August 21, 1981; in revised form October 25, 1981

Note added in proof. Recently T. Geisel and J. Nierwetberg (Phys. Rev. Lett. 47, 975, 1981) presented results which agree with the ones proved in the present paper. They generalize the procedure to sequences $q 2^{k}, k$ fixed, take the limit $k \rightarrow \infty$, and find interesting universal behaviour. The curves in their Fig. 1 are examples satisfying, in the $q \rightarrow \infty$ limit, our scaling $h(x)$ and coincide with each other if plotted as functions of $\chi \equiv \Delta^{q}(1-\mu)$. 